(revised $27^{\text {th }}$ September 2013)

\title{
Personal resilience in times of crisis: The implications of SWB homeostasis and set-points \\ Robert A. Cummins ${ }^{1}$ \\ Mark Wooden ${ }^{2}$
}

\author{
${ }^{1}$ School of Psychology, Deakin University \\ ${ }^{2}$ Melbourne Institute of Applied Economic and Social Research, University of Melbourne
}

\begin{abstract}
Our companion paper (Cummins, Li, Wooden, \& Stokes, 2013) describes the statistical process used to demonstrate set-points and set-point-ranges for subjective wellbeing. The implications of set-points and homeostasis are now considered in the context of resilience. This discussion leads with a brief overview of resilience definitions and is followed by a description of SWB homeostasis. This addresses, in particular, the issue of SWB malleability under homeostatic control. The link between resources and resilience is then considered, in terms of predictions made by homeostasis theory. Finally, discussion focuses on the implications of such understanding for future directions in SWB research. It is concluded that an understanding of set-points and homeostasis allows new insights into the resilience construct.
\end{abstract}

\begin{abstract}
Our companion paper (Cummins, Li, Wooden, \& Stokes, 2013) describes the statistical process used to demonstrate set-points and set-point-ranges for subjective wellbeing. The implications of set-points and homeostasis are now considered in the context of resilience. This discussion leads with a brief overview of resilience definitions and is followed by a description of SWB homeostasis. This addresses, in particular, the issue of SWB malleability under homeostatic control. The link between resources and resilience is then considered, in terms of predictions made by homeostasis theory. Finally, discussion focuses on the implications of such understanding for future directions in SWB research. It is concluded that an understanding of set-points and homeostasis allows new insights into the resilience construct.
\end{abstract}

\section{Introduction}

\section{Defining resilience}

The starting point for this discussion must be the definition of 'resilience', since there is such a diversity of opinion. Perhaps the most generic definition comes from Merriam-Webster (2012) as:

'an ability to recover from or adjust easily to misfortune or change'.

This is clever because it is non-specific in all respects. It does not specify the process of recovery or adjustment, the nature of the misfortune or change, or the temporal dimension. Thus, in relation to the latter, it is equally applicable to the resilience of children raised in adverse circumstances (Werner \& Smith, 1982) and to adults faced with a life crisis. Other 
dictionary definitions are generally more specific and, as a result, are more exclusive. For example, the Macmillan Dictionary (2012) defines resilience as:

'Someone's ability to become healthy, happy, or strong again after an illness, disappointment, or other problem.'

This excludes children raised in adverse circumstances or adults who have incurable cancer at the time of an additional challenging event, and limits the dimensions of recovery to health, happiness and strength. It thereby omits forgiveness as a dimension of resilience, for example.

Other uses of 'resilience' reflect the orientation of the source authors. Thus a focus exclusively on children is common; for example, "a process or phenomenon reflecting positive child adjustment despite conditions of risk" (Luthar \& Zelazo, 2003, p. 510). Again, all such definitions limit their application.

Academic definitions often attempt to provide understanding of the resilience process. Thus, for example, Wilson and Ferch (2005) emphasise qualities of mental flexibility and creativity:

"Resilience refers to the psychological ability to let go of old internal structures of thinking and behaving - to create and reintegrate new structures - that provide us a more mature sense of coherence" (p. 48).

Such tight specification is a long way from generic. In this case it discounts someone who has already learned the skills and gathered the resources to be resilient in a crisis. It also omits the use of financial resources, for example, to employ other people to deal with the crisis. This and many of the other definitions that can be found (e.g., Peterson, Balthazard, Waldman, \& Thatcher, 2008) betray the authors' research orientation, rather than providing a broad base of understanding.

The definition that will be used to guide the discussion to follow is the broad, generic view offered by Merriam-Webster (2012), as an ability to recover from, or adjust easily to, misfortune or change.

\section{Resilience and domains of attraction}

Perhaps the grandest context for the discussion of resilience is in relation to ecological systems, and a thoughtful discussion has been provided by Holling (1973). He proposes that resilience reflects the persistence of intra-system relationships. It is the ability of an open system (a system which continuously interacts with its environment or surroundings) to absorb the changes caused by impinging variables, and survive. Thus, the resilience of an ecological system is the inverse probability of its extinction.

In this view, each such system has a theoretical boundary which marks the area of stable interactive relationships. This region, within which stability occurs, is called the 'domain of attraction'. Within this theoretical boundary, all possible variable trajectories spiral outward and inward to create a state of dynamic equilibrium around a point of neutrality. However, once outside the domain boundary, variable trajectories continue to spiral outwards leading to the extinction of the variable, and possibly of the system. 
Such systems also have a property called 'stability', which is the speed with which a system returns to equilibrium after a temporary disturbance. High stability is characteristic of benign climatic circumstances. Here the domain boundaries tend to be small and the populations stable, but with low resilience. Thus, they are prone to extinction when exposed to chance climatic extremes. In ecological areas subject to extreme climatic conditions, the reverse applies. Here populations can fluctuate widely, and so be unstable, but also have high resilience in that the ecological system survives.

It is important to note that there is no single attraction domain or constant level of equilibrium, even in relation to a single ecological system. For example, in response to prolonged environmental deterioration, the point of neutrality may shift downward, to create a new domain of attraction.

This conception of resilience has parallels in psychology. Dynamic Systems Theory (e.g. Mahoney, 1991; Vallacher \& Nowak, 1997) conceptualizes the behavior of a system over time as occurring within an 'attractor' region. In terms similar to ecological systems, it is proposed that a set of operations are contained within this region by a metaphoric 'gravitational force'. Such attractor regions also have varying degrees of stability, and they also have 'optimality', conceived as the degree of optimal adaptation, or resilience. This property of optimality allows the statement of broad goal of therapy, which is to move the person from a sub-optimal region to one that is more optimal. However, since no empirical measures are involved, the described system is highly figurative. In a slightly more empirical sense, Carver (1998) uses 'attractor region' to stand for the person's overall life space and their total adaptation to the circumstances of their life. Similarly, Seligman (2002) uses adaptation as the returning force for affective states contained within a 'set-range' bounded by a metaphorical 'permeable membrane'.

While such concepts are useful philosophical devices, more concrete and measurable conceptions of resilience have been generated within psychology.

\section{Resilience in Psychology}

Comprehension of the hypothetical resilience construct in psychology is impeded by the use of poorly defined and overlapping terms. Multiple authors have expressed their views within a terminological and conceptual jungle. There are even synonyms for resilience which include 'positive adaptation' (Conger \& Conger, 2002) and the 'ability to see reality' (Antonovsky, 1979), which offer their own particular brands.

However, at a global, conceptual level, researchers seem to generally agree that resilience can exist within the Merriam-Webster (2012) definition. Consequently, it is most commonly considered that the processes of recovery and adjustment must require resources of some kind. It therefore follows that, ceteris paribus, more resources will yield more resilience. However, describing the nature of such resources, in the most parsimonious and theoretically useful way, has yielded much diversity of opinion.

At the most generic level, such resources have been described as 'generalized resilience resources' (Antonovsky, 1979), 'regulatory capital' (Masten \& Coatsworth, 1998), 'buffers' (Ensel \& Lin, 1991; Werner, 1995), 'hedonic capital' (Graham \& Oswald, 2010), 'psychological wellbeing' (Ryff \& Singer, 1998), and 'ego-resilience' (Block \& Kremen, 1996). Each term is used to signal a different theoretical approach to describing resilience, 
which could be useful if the authors delineated how, precisely, their own resource terms differ from those of others. But, inevitably, they do not, and there is much overlap.

At the next level of resource specificity there are such terms as self-concept clarity (Campbell, 1990), self-esteem (Taylor \& Stanton, 2007), optimism (Flach, 1988); control and coping (Conger \& Conger, 2002; Ensel \& Lin, 1991; Fernández-Rios \& Novo, 2012); personality (Ramanathan, Wardecker, Slocomb, \& Hillary, 2011) and positive affect (Diener \& Chan, 2011). All of the resources at this level have scales of measurement, and all of them produce data that automatically correlate with one another due to the shared variance of Homeostatically Protected Mood (see later). An extended list of these positive psychological resources is provided by Masten (2007) and is dominated by items that can be generally categorized as either personal relationships or perceived control.

The feeling of being meaningfully connected to at least one other person, and that one's life is under control, are undoubtedly central resilience resources. The importance of such resources appears to cross cultural and age demographics (e.g., Werner, 1995). Both of these resilience constructs are also clearly influenced by personality and, therefore, by genetics. This link is most obvious through extraversion, which is strongly connected to both relationships and control. As observed by a number of authors (e.g. Carver, 1998; Conger \& Conger, 2002), the sense of belonging and being in control has a double benefit for resilience. Both link to positive affect and the probability that future sources of stress will be minimized due to active intervention.

Finally, there are the terms used to describe the outcome of successful resilience, such as wellbeing (Hamilton, 2006), thriving (Carver, 1998), reaching potential, and flourishing (Seligman, 2011). The data from scales that operationalize these terms are crucial to defining resilience. This is because variation in such outcome measures is interpreted as being consequential to the operation of some set of defined independent variables, which are then used to infer the mechanism of resilience.

In summary, there is a huge literature on resilience within psychology. Much of it is repetitive and much of it concerns the positive interactions between the specific resource variables mentioned above. Various theoretical schemes have been proposed to give an overarching theoretical view of resilience, and some have been listed earlier. Another is the Theory of Subjective Wellbeing Homeostasis which incorporates both the idea of 'attractors' and the non-linear relationships of Dynamic Systems Theory.

\section{Homeostasis theory}

Subjective Wellbeing (SWB) Homeostasis theory asserts that each individual has a set-point for their SWB which is a genetically-determined individual difference. In the companion paper (Cummins et al., 2013) SWB is measured by using the question 'All things considered, how satisfied are you with your life?' (General Life Satisfaction: GLS) rated on a $0-10$ response scale. All data are then projected onto a $0-100$ point scale. In that paper, we find that set-points have a normal population distribution of 70 to 90 points positive, with an average of 80 .

We also propose that SWB is saturated with a form of affect we call 'Homeostatically Protected Mood' (HPMood: Cummins, 2010). Inspired by Russell (2003), HPMood is conceived as a neuro-physiologically generated affect comprising the simplest, constant, nonreflective feeling. It is not modifiable by conscious experience, yet is a ubiquitous component 
of conscious experience. It is this steady-state, affective set-point, that homeostasis seeks to defend.

Support for the idea that HPMood dominates the composition of SWB was initially provided by Davern, Cummins, and Stokes (2007), who represented HPMood by the three affects of 'happy', 'contented' and 'excited'. They found these affects together explained $64 \%$ of the variance in SWB. This domination of SWB by HPMood has been confirmed by Blore, Stokes, Mellor, Firth, and Cummins (2011) and Tomyn and Cummins (2011). These authors suggest that HPMood is the driving force behind SWB, not personality as is generally reported in the literature (e.g. DeNeve \& Cooper, 1998; Emmons \& Diener, 1985; Headey \& Wearing, 1992; Vitterso \& Nilsen, 2002).

We also propose that, while the affect generated as HPMood is constant, our conscious experience of affect is highly variable. Such conscious awareness comprises a combination of HPMood and whatever emotions are being generated by momentary experience. These emotions may well be a stronger source of affect than HPMood, thereby causing experienced affect to be different from set-point. When this occurs, we propose, homeostatic forces are activated to return experienced affect to its set-point. Thus, actual affective experience normally oscillates around its set-point and, as long as homeostasis is in control, affective experience remains within its set-point-range.

When SWB moves outside its set-point-range, homeostatic stabilizing forces are activated which include behaviour, adaptation, and a system of cognitive buffers. The first of these is self-evident; behaviour can be used to either engage or disengage from an emotionally intense situation. The topic of adaptation has been covered in the companion paper, so this leaves the cognitive buffers (see Cummins, 2010 for an extended description). These are those same constructs used by Taylor and Brown (1988) as evidence of positive distortion in self-evaluation. They are self-esteem (i.e. feelings of self-worth; Cummins \& Nistico, 2002), perceived control (i.e. perception that that one can achieve desired outcomes through their own actions Thompson, Armstrong, \& Thoman, 1998) and optimism (i.e. the belief that one's future is bright; Peterson, 2000).

These positive buffers are in intimate, conscious interaction with momentary experience. They are strongly perfused with positive-activated mood (HPMood), which is delivered at the level of each set-point. They also have a cognitive component which is responsible for adjusting each buffer, as required, in order to defend HPMood. Thus, for example, a failure of secondary control (Rothbaum, Weisz, \& Snyder, 1982) that causes SWB to fall may be counteracted by thinking that the failure experience was trivial in the grand scheme of life. In summary, Homeostasis theory proposes these three constructs as psychological buffers because they have a role in protecting the positive-activated affect generated as HPMood.

All of these psychological devices used to maintain homeostasis are reinforced by external buffers, the most powerful of which are an intimate relationship and income. A huge literature attests to the efficacy of these variables in reducing the power of potential stressors. They help defend HPMood by utilizing social support or the purchase of assistance (Cummins, in press). Importantly for an understanding of resilience, the relationship between these two buffers and psychological resources is normally bi-directional (e.g. Ensel \& Lin, 1991). Strong psychological resources are likely to garner strong external resources, thereby enhancing resilience. The reverse will also, of course, apply. 
Whether, and for how long, a particular challenge will move experienced affect outside each set-point-range will depend on the strength of the challenging agent matched against resilience. Resilience in this context is the sum of the protective resources described above. Provided that resilience is sufficiently strong, SWB may either continue to be maintained within its normal range or will leave the range briefly. However, if the strength of the challenge exceeds the capacity of resilience to counter such challenge, the homeostatic system will forfeit control to the challenging agent, resulting in either a drop or a rise in SWB beyond its set-point-range (for an elaboration of these propositions see Cummins, 2010).

The description above is similar to that suggested by Carver (1998), who proposed that the term resilience be used to denote homeostatic return to a prior condition. However, his explanation of recovery is limited to adaptation and conceives a return to a baseline, not to a set-point.

\section{Theoretical and empirical cohesion}

Homeostasis theory offers a high level of conceptual cohesion in understanding the commonly observed phenomenon that SWB levels display resilient stability (Graham \& Oswald, 2010). Calling on the known properties of homeostatic systems, it makes highly specific predictions concerning the empirical relationships between SWB, challenging agents, and protective resources. The predictive model is shown in Figure 1.

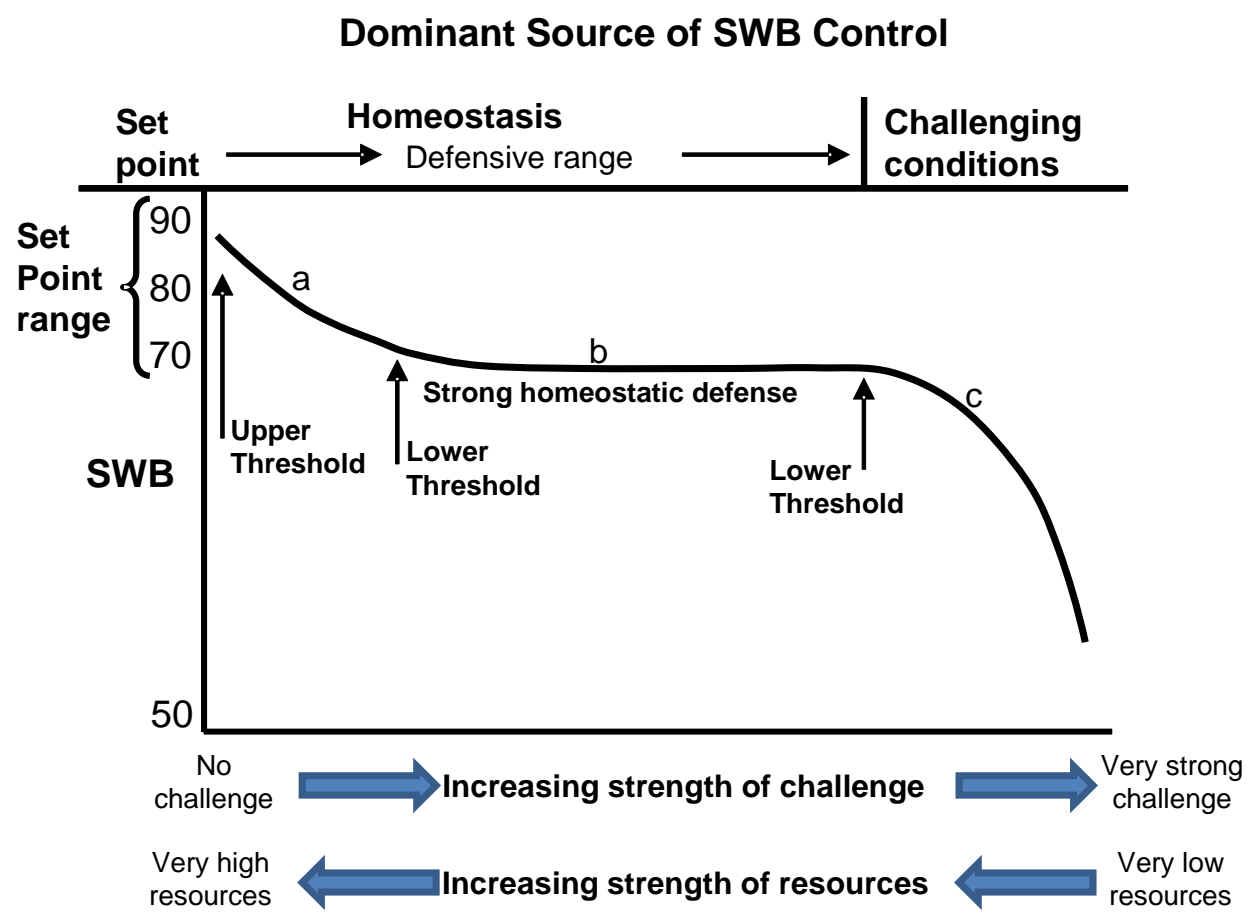

Figure 1: Changing levels of SWB as homeostasis is challenged.

The interpretation of Figure 1 is as follows:

1. The vertical axis depicts the 0-100 scale of SWB and shows the normal distribution of set-points ranging from 70 to 90 points (Cummins et al., 2013). 
2. The lower horizontal axis shows the two competing forces involving resilience. One is the strength of a negative challenge to SWB, such as might be delivered by poverty or anxiety. The other is the strength of homeostatic resources, such as from income or an intimate relationship.

3. The upper horizontal axis shows the dominant source of control. The source of control changes depending on the balance between the strength of challenge and the strength of resilience resources.

4. The curved 'response line' depicts a mid-range set-point of 80 and an assumed (not shown) set-point-range of 20 points. As explained in Cummins et al. (2013), this range magnitude is almost certainly an over-estimation. Coincidentally, in this example, the magnitude of the set-point-range coincides with the range of set-points. Changes in the level of SWB are due to changes in the challenge/resources balance. The upper (90 point) and lower (70 point) margins of the set-point-range are identified as the setpoint-range 'thresholds' and indicated by vertical arrows.

The relationships depicted in Figure 1 can be used to make various predictions regarding the non-linear relationship between SWB and resources. Some of these are as follows:

1. Under conditions of zero challenge, SWB will average to its set-point, which for this individual is 80 points. Thus, the group average for people living under such nonthreatening conditions will also be 80 points.

2. As mild sources of threat are experienced, the level of SWB will vary within its setpoint-range. Moreover, its position within its set-point-range will be a probability statement determined by the balance of good and bad momentary experience and the resilience of the homeostatic system. Thus, a sustained, supportive environment, where good experience dominates, will allow SWB to average within the upper portion of the set-point range, depicted as (a) in Figure 1. A sustained challenging environment, where negative experience dominates, will cause SWB to average within the lower portion of the set-point-range, depicted as (b).

3. As the strength of challenge intensifies, the strength of homeostatic defence also increases in an attempt to maintain stable levels of SWB. The result is the prolonged phase (b), in which homeostasis manages to hold the line and prevents SWB from decreasing below its lower threshold value of 70 points. The evidence for this lower threshold to be located at about 70 points, on average, is presented in Cummins (2003).

Importantly during this phase (b), the value of SWB is insensitive to changing levels of the challenging agent. That is, although the strength of the challenge is increasing, SWB will be held steady at the value above the lower threshold. This phase will continue as long as the homeostatic system is effective. However, at some higher strength of challenge, homeostasis will be overwhelmed.

4. Once the strength of the challenging agent becomes too strong for homeostatic management, the value of SWB enters phase (c). In this phase the dominant source of control has shifted from homeostatic processes to the challenging agent. This has been demonstrated for the relationship between SWB and increasing levels of obesity (Cummins, 2012). 


\section{Predictions arising from the homeostatic model}

Almost inevitably in the empirical literature, researchers study the relationship between levels of SWB and other variables using linear statistics. That is, they assume they are dealing with simple relationships such that, for example, increased income will induce increased SWB and increased anxiety will induce decreased SWB. But homeostasis theory (Cummins, in press) predicts otherwise.

Homeostasis theory makes the following predictions:

1. Under normal operating conditions where SWB is under homeostatic control, the presence of either a positive or negative challenge, of the minor sort people encounter each day, has very little effect on SWB. Any small perturbations will quickly be corrected by homeostatic processes, and SWB will return to its set-point range. The stability of SWB over various time periods has been well documented (Diener, Inglehart, \& Tay, 2013).

2. In the face of a major and sustained challenge, the effect on SWB will depend on its initial relationship to its set-point range (Tomyn, Weinberg, \& Cummins, 2013). If the challenge is positive, there are three scenarios, each one dependent on the initial level of SWB as follows:

2.1 If SWB is in the positive half of its range, then the effect will be minimal. SWB may well rise above its set-point-range as an acute reaction, but adaptation and habituation will ensure a fairly rapid return to range. The chronic effect will be minimal because SWB was initially in the upper portion of its range and can be held no higher.

2.2 If SWB is in the negative half of its range, the chronic influence to lift SWB will still be small, but probably measurable (Engel \& Cummins, 2011). If the positive resource is sufficiently strong to maintain SWB at a higher level than it was, then the degree of increase will depend on the extent of sustained movement within the range, and this is uncertain. While we estimate that the total extent of each set-point-range is a maximum of 20 points, this is almost certainly a considerable over-estimation of the degree of sustained change while maintaining homeostatic control. Not only is the range likely too large (Cummins et al., 2013) but also it is not known how close to the edge of each range SWB can be maintained. Quite probably, SWB stability is inversely proportional to its distance from set-point. All in all, it seems unlikely that circumstances could reliably increase SWB by more than a few percentage points while under homeostatic control.

2.3 If SWB is in homeostatic defeat, then very large increases in SWB are possible. The potential magnitude of change is the distance between the starting level and the upper portion of the set-point-range. Under conditions of negative homeostatic defeat, the value of SWB becomes highly sensitive to the strength of homeostatic resources, such as income, relationships or an effective intervention (Cummins, Hammond, \& Campbell, 2012a).

3. If the challenge is negative, then there are two scenarios. However, these differ from those described above in relation to positive challenge. This is because there is no ceiling effect and a very uncertain floor effect. In the face of a negative challenge, the 
extent of SWB decrease will depend on three factors. The first is the initial level of SWB, the second is the strength of homeostatic resilience, and the third is the cumulative level of challenge. That is, the power of a specific negative event to challenge homeostasis is cumulative with other negative experiences that are present at the same time.

3.1 If SWB is in the positive half of its range and the cumulative level of challenge is not sufficient to defeat homeostasis, then the situation is similar to that described under 2.2 above. The effect will be small but probably measurable. If, however, the cumulative challenge is sufficiently strong to defeat homeostasis, then the control of SWB passes from homeostasis to the challenging agents, and SWB falls below its range. Once it has done this, it may or may not return to lie within its set-point-range. Whether it does so, and over what time span it does so, will depend on the balance of power between the resilience of homeostatic forces and the cumulative power of the challenge.

3.2 If SWB is already below its set-point-range, then homeostatic resilience is no longer effective. Under such conditions, SWB will be highly sensitive to an increase in the level of resources, but probably less sensitive to additional levels of challenge.

4. Homeostatic resilience is strengthened by the behavioural, external and internal resources described earlier. All of these resource types have limitations in the extent to which they can be used as a positive, adaptive resource for resilience. Understanding the reason for this is crucial to understanding how resilience may be strengthened. There are two principles. First, each form of resource has an optimal capacity and, second, more of a particular resource is not necessarily better and may actually damage effective adaptation. The three sources of resource are now separately considered within this context:

4.1 The effect of using a simple behaviour as a resilience resource, such as disengaging from the source of challenge, is effective only if such disengagement is actually adaptive. It may be so when faced with a verbally abusive stranger. However, caring for a disabled family member is very likely to be challenging and, for many people, so strongly challenging that it defeats homeostasis (Cummins et al., 2012a). In such a situation, simple disengagement from the caring task will likely have severe consequences. Moreover, the general strategy of disengaging from challenge is adaptive only to a finite degree. An over-use of disengagement may cause ineffective engagement with the normal world of interpersonal relationships.

A similar argument can be made in relation to the use of any other, single behavioural strategy, such as active confrontation, physical submission, friendly demeanour, etc. For all of these, the strategy needs to be used with discretion in order to be optimally useful as a homeostatic resource.

4.2 The effect of increasing the strength of the cognitive buffers may, or may not, be an adaptive strategy. Take, for example, optimism. Having a robust level of optimism is generally adaptive. However, the extremes of optimism are clearly maladaptive. Very low levels associate with fragile resilience too easily defeated, while very high levels can give rise to impulsive choices in complex situations. 
Since optimism is heavily saturated with HPMood (Lai \& Cummins, 2012), it is likely that the most generally adaptive level is 80 points, since this is the midpoint of the set-point distribution (Cummins et al., 2013). However, this may well change under circumstances where the outcome is optimised by making more careful, or less careful, choices. Deciding when to sow a crop is advantaged by low optimism, in that low positive affect facilities careful weighing of the available evidence (Forgas, 2008). In the heat of battle, on the other hand, deciding which parry or thrust is advantageous requires instantaneous judgement.

A similar argument can be made in relation to the other cognitive buffers of self-esteem and perceived control. Again, 80 points is likely to be generally optimal, but more, or less, may be better under particular circumstances.

4.3 The situation regarding the external resources of relationships and income is rather different. Here, the adaptive value of increasing each resource will depend on the degree to whether homeostatic resilience is already saturated with that resource. If someone is low on income, then acquiring more money can strengthen resilience. However, if people are wealthy then increasing their wealth will be unlikely to increase their resilience. For example, if their major source of challenge is the emotional pain of bereavement, and the person is already receiving expert professional assistance to deal with this issue, then more income will be ineffective. Similarly, for someone who has an emotionally intimate relationship, adding another source of emotional intimacy may decrease the effectiveness of their existing relationship.

Once again, there is an optimal level of each external resource. Increasing the strength of the resource for people with levels that are too low will be beneficial. Increasing the levels for people whose homeostatic resilience is already saturated with the resource is at best ineffective and at worst harmful.

The way these propositions can be tested is through the segmentation of large samples into sub groups based on their initial level of SWB. Ideally this separation can be done into deciles, as demonstrated by Tomyn et al. (2013). However, this is impractical with smaller samples which do not have sufficient $\mathrm{N}$ to allow results based on such sub-groups to be reliable. Smaller samples can be separated into ranges which are functionally consistent with the companion article on set-points. That is, sub-samples comprising individuals with a SWB above 70 points contain people who are in normal homeostatic maintenance; sub-samples based on scores between 50 and 70 points contain a mixture of people maintaining homeostasis and people in homeostatic failure; while sub-samples based on people with an SWB below 50 points contain people in homeostatic failure.

\section{Summary and implications of homeostatic resilience}

It has been argued that, for people living in favorable environments, the strongest determinant of mood happiness is their genetic set-point. Thus, for samples comprising such people, the sample variance will be dominated by the distribution of set-points. Moreover, this distribution is invariant (normal) and not subject to influence by external factors, such as wealth or health. This domination of sample variance by set-points adds a dampening and stabilizing factor to the mean SWB of such samples. It is for this reason that the SWB level of normal population samples in Australia is quite hard to change (Cummins et al., 2012c). The second strongest determinant of mood happiness is resilience. 
In this context, resilience is the power of homeostasis, first to retain control of SWB and, second, to restore the dominance of HPMood following excursion of the affective experience outside the 'attractor region' (set-point-range). As a consequence of retained homeostatic control, and provided that respondents are operating within their set-point-range at baseline, the addition of weak resources or challenges to their life will normally have little impact on measured levels of SWB. Due to the restorative power of homeostatic resilience, affective excursions beyond the set-point-range will be short-lived. However, under chronic challenge by a strong emotional experience, homeostatic resilience fails (Cummins et al., 2007)

When homeostasis fails to retain control of SWB, people's responses to SWB items will be dominated by affect generated by the challenging agent (Cummins, Lau, \& Davern, 2012b). Under such conditions, as has been previously discussed, SWB will be highly sensitive to the power of resources and challenges since homeostasis is no longer operating as an effective buffering agent.

This understanding has considerable implications for the study of relationships between SWB, resources, and challenges. Most important, it predicts that the degree of change in SWB due to some positive resource or some negative challenge will be exquisitely sensitive to baseline levels. If SWB is within normal range at baseline, then it will appear more rigid than fluid. If, however, SWB at baseline is below normal range, then theory predicts that SWB will show considerable sensitivity to resources.

There are three further implications that derive from the idea of homeostatic resilience. Each has significant implications for possible future research.

The first concerns the connection between set-points and resilience. It has been commonly assumed in the literature that people with higher SWB have higher resilience (e.g., Graham \& Oswald, 2010). If this is so, then it may follow that people with high set-points are intrinsically more resilient. Since HPMood is proposed to be driving levels of the homeostatic buffers (self-esteem, optimism and perceived control) (Cummins, 2010) then it might be expected that higher levels of these buffers equates to more resilience.

The second implication concerns social policy. This arises from the mismatch between population SWB and 80 points. That is, from (Cummins et al., 2012c), the population mean for GLS, averaged over 26 surveys in Australia, is 77.65 points. Yet the theoretical population mean should be 80 points according to the distribution of set-points (Cummins et al., 2013). Thus, the population mean is about 2.3 points lower than it would be if all respondents were operating at their set-point.

What this surely represents is a discounted population mean caused by inclusion of respondents suffering homeostatic challenge and defeat. This causes people to report GLS levels that lie either in the lower portion of their set-point-range, or actually below this range due to outright homeostatic failure. No doubt some of this defeat could be rectified by the allocation of additional financial or relationship resources. Thus, in terms of national policy concerning the distribution of financial resources, at least, these 2.3 percentage points can be considered aspirational. This gap represents the theoretical increase in national SWB which could be achieved by the distribution of additional resources to people in need.

The third and final implication concerns the nature of what is measured within the ambit of SWB research. The above analysis provides at least a partial explanation for why the average SWB level of general population samples is so resistant to change in economically mature 
countries (see e.g., Cummins et al., 2012c - Chapter 2). But studying changed mean scores may be missing a more powerful effect. The importance of adding resources to normally functioning populations may not be found in raised levels of SWB, but rather in the increased homeostatic resilience such resources confer.

\section{References}

Antonovsky, A. (1979). Health, stress, and coping. San Francisco: Jossey-Bass.

Block, J., \& Kremen, A. M. (1996). IQ and ego-resiliency: conceptual and empirical connections and separateness. Journal of Personality and Social Psychology, 70, 349361.

Blore, J. D., Stokes, M. A., Mellor, D., Firth, L., \& Cummins, R. A. (2011). Comparing multiple discrepancies theory to affective models of subjective wellbeing. Social Indicators Research, 100(1), 1-16. doi: 10.1007/s11205-010-9599-2

Campbell, J. D. (1990). Self-esteem and clarity of the self-concept. Journal of Personality and Social Psychology, 59, 538-549.

Carver, C. S. (1998). Resilience and thriving: Issues, models, and linkages. Journal of Social Issues, 54, 245-266.

Conger, R. D., \& Conger, K. J. (2002). Resilience in midwestern families: Selected findings from the first decade of a prospective, longitudinal study. Journal of Marriage and Family, 64, 361-373.

Cummins, R. A. (2003). Normative life satisfaction: Measurement issues and a homeostatic model. Social Indicators Research, 64(2), 225-256.

Cummins, R. A. (2010). Subjective wellbeing, homeostatically protected mood and depression: A synthesis. Journal of Happiness Studies, 11, 1-17. doi: 10.1007/s10902009-9167-0

Cummins, R. A. (2012). The relationship between subjective wellbeing and health. In M. L. Caltabiano \& L. A. Ricciardelli (Eds.), Applied topics in health psychology (pp. 101111). West Sussex, UK: John Wiley \& Sons.

Cummins, R. A. (in press). Subjective Wellbeing Homeostasis. In D. S. Dunn (Ed.), Oxford Bibliographies in Psychology. Oxford University Press New York. 
Cummins, R. A., Hammond, T., \& Campbell, P. (2012a). Carers Counselling Intervention Study - Volume 2. Melbourne: Australian Centre on Quality of Life, School of Psychology, Deakin University. ISBN 978-1-74156-162-3.

Cummins, R. A., Hughes, J., Tomyn, A., Gibson, A., Woerner, J., \& Lai, L. (2007). Australian Unity Wellbeing Index: Report 17.1 The Wellbeing of Australians - Carer Health and Wellbeing. Retrieved April 8, 2013, from http://www.deakin.edu.au/research/acqol/index_wellbeing/index.htm

Cummins, R. A., Lau, A. D. L., \& Davern, M. (2012b). Subjective wellbeing homeostasis. In K. C. Land, A. Michalos \& J. Sirgy (Eds.), Handbook of social indicators and quality-of-life studies. Volume I: Theoretical and Methodological Foundations (pp. 79-98). New York: Springer.

Cummins, R. A., Li, L., Wooden, M., \& Stokes, M. (2013). A demonstration of set-points for subjective wellbeing. Journal of Happiness Studies. doi: 10.1007/s10902-013-9444-9

Cummins, R. A., \& Nistico, H. (2002). Maintaining life satisfaction: The role of positive cognitive bias. Journal of Happiness Studies, 3(1), 37-69.

Cummins, R. A., Woerner, J., Weinberg, M., Collard, J., Hartley-Clark, L., Perera, C., \& Horfiniak, K. C. () . . . (2012c). Australian Unity Wellbeing Index: - Report 28.0 The Wellbeing of Australians -The impact of marriage. Retrieved April 8, 2013, from http://www.deakin.edu.au/research/acqol/index_wellbeing/index.htm

Davern, M., Cummins, R. A., \& Stokes, M. (2007). Subjective wellbeing as an affective/cognitive construct. Journal of Happiness Studies, 8(4), 429-449. doi: 10.1007/s10902-007-9066-1

DeNeve, K. M., \& Cooper, H. (1998). The happy personality: A meta-analysis of 137 personality traits and subjective well-being. Psychological Bulletin, 124, 197-229.

Diener, E., \& Chan, M. Y. (2011). Happy people live longer: Subjective well-being contributes to health and longevity. Applied Psychology: Health and well-being, 3(1), 1-43. doi: 10.1111/j.1758-0854.2010.01045.x

Diener, E., Inglehart, R., \& Tay, L. (2013). The validity of life satisfaction measures. Social Indicators Research, 112(3), 497-527. doi: 10.1007/s11205-012-0076-y

Emmons, R. A., \& Diener, E. (1985). Personality correlates of subjective well-being. Personality and Social Psychology Bulletin, 11, 89-97. 
Engel, L., \& Cummins, R. A. (2011). Impact of dose adjustment for normal eating in Australia (OzDAFNE) on subjective wellbeing, coping resources and negative affects in adults with type 1 diabetes: A prospective comparison study. Diabetes Research and Clinical Practice,, 91, 271-279. doi: 10.1016/j.diabres.2010.11.023

Ensel, W. M., \& Lin, N. (1991). The life stress paradigm and psychological distress. Journal of Health and Social Behavior, 32, 321-341.

Fernández-Rios, L., \& Novo, M. (2012). Positive psychology: Zeigeist (or spirit of the times) or ignorance (or disinformation) of history? . International Journal of Clinical and Health Psychology, 12(2), 333-344.

Flach, F. (1988). Resilience: Discovering a new strength at times of stress. New York: Fawcett Columbine.

Forgas, J. P. (2008). The strange cognitive benefits of mild dysphoria: On the evolutionary advantages of not being too happy. In J. P. Forgas, M. G. Haselton \& W. von Hippel (Eds.), Evolutionary psychology and Social cognition (pp. 107-121). New York: Psychology Press.

Graham, L., \& Oswald, A. J. (2010). Hedonic capital, adaptation and resilience. Journal of Economic Behavior \& Organization, 76(2), 372-384.

Hamilton, C. (2006) What's left? The death of social democracy. Quarterly Essay, Vol. 21. Melbourne: Black Inc.

Headey, B., \& Wearing, A. (1992). Understanding happiness: A theory of subjective wellbeing. Melbourne: Longman Cheshire.

Holling, C. S. (1973). Resilience and stability of ecological systems. Annual Review of Ecological Systems, 4, 1-23.

Lai, L.C. H., \& Cummins, R. A. (2012). The contribution of job and partner satisfaction to the homeostatic defense of subjective wellbeing. Social Indicators Research doi: 10.1007/s11205-011-9991-6

Luthar, S. S., \& Zelazo, L. B. (2003). Research on resilience: An integrative review. In S. S. Luthar (Ed.), Resilience and vulnerability: Adaptation in the context of childhood adversities (pp. 511-549). Cambridge, UK: Cambridge University Press.

Macmillan Dictionary. (2012). Retrieved October 25, 2012, from http://www.macmillandictionary.com/dictionary/american/resilience 
Mahoney, M. J. (1991). Human change processes: The scientific foundations of psychotherapy. New York: Basic Books.

Masten, A. S. (2007). Resilience in developing systems: progress and promise as the fourth wave rises. Development and Psychopathology, 19, 921-930.

Masten, A. S., \& Coatsworth, J. D. (1998). The development of competence in favorable and unfavorable environments: Lessons from research on successful children. American Psychologist, 53, 205-220.

Merriam-Webster. (2012). Retrieved October 25, 2012, from http://www.merriamwebster.com

Peterson, C. (2000). The future of optimism. American Psychologist, 55, 44-56.

Peterson, S. J., Balthazard, P. A., Waldman, D. A., \& Thatcher, R. W. (2008). Neuroscientific implications of psychological capital: Are the brains of optimistic, hopeful, confident, and resilient leaders different? Organisational Dynamics, 37, 342-353.

Ramanathan, D. M., Wardecker, B. M., Slocomb, J. E. , \& Hillary, F. G. (2011). Dispositional optimism and outcome following traumatic brain injury. Brain Injury, 25(4), 328-337.

Rothbaum, F., Weisz, J. R., \& Snyder, S. S. (1982). Changing the world and changing the self: A two-process model of perceived control. Journal of Personality and Social Psychology, 42(1), 5-37.

Russell, J. A. (2003). Core affect and the psychological construction of emotion. Psychological Review, 110(1), 145-172.

Ryff, C. D., \& Singer, B. H. (1998). The contours of positive human health. Psychological Inquiry, 9(1-28). doi: org/10.1207/s15327965pli0901_1

Seligman, M. E. P. (2002). Authentic Happiness: Using the new positive psychology to realise your potential for lasting fulfillment. New York: The Free Press.

Seligman, M.E.P. (2011). Flourish: A Visionary New Understanding of Happiness and Wellbeing. New York: Simon \& Schuster.

Taylor, S. E., \& Brown, J. D. (1988). Illusion and well-being: A social psychological perspective on mental health. Psychological Bulletin, 103, 193-210. 
Taylor, S. E., \& Stanton, A. L. (2007). Coping resources, coping processes, and mental health. Annual Review of Clinical Psychology, 3, 377-401.

Thompson, S. C., Armstrong, W., \& Thoman, C. (1998). Illusions of control, underestimations, and accuracy: A control heuristic explanation. Psychological Bulletin, 123, 143-161.

Tomyn, A. J., \& Cummins, R. A. (2011). Subjective wellbeing and homeostatically protected mood: Theory validation with adolescents. Journal of Happiness Studies, 12(5), 897914. doi: 10.1007/s10902-010-9235-5

Tomyn, A. J., Weinberg, M. K., \& Cummins, R. A. (2013). Intervention efficacy among 'at risk' adolescents: A test of Subjective Wellbeing Homeostasis Theory. Social Indicators Research.

Vallacher, R. R., \& Nowak, A. (1997). The emergence of dynamical social psychology. Psychological Inquiry, 8, 73-99.

Vitterso, J., \& Nilsen, F. (2002). The conceptual and relational structure of subjective wellbeing, neuroticism, and extraversion: Once again, neuroticism is the important predictor of happiness. Social Indicators Research, 57 (1), 89.

Werner, E. E. (1995). Resilience in development. Current Directions in Psychological Science, 3, 81-85.

Werner, E. E., \& Smith, R. S. (1982). Vulnerable but invincible: A study of resilient children. New York: McGraw-Hill.

Wilson, S., \& Ferch, S. (2005). Enhancing resilience in the workplace through the practice of caring relationships. Organisation Development Journal, 23, 45-60. 


\section{Dominant Source of SWB Control}

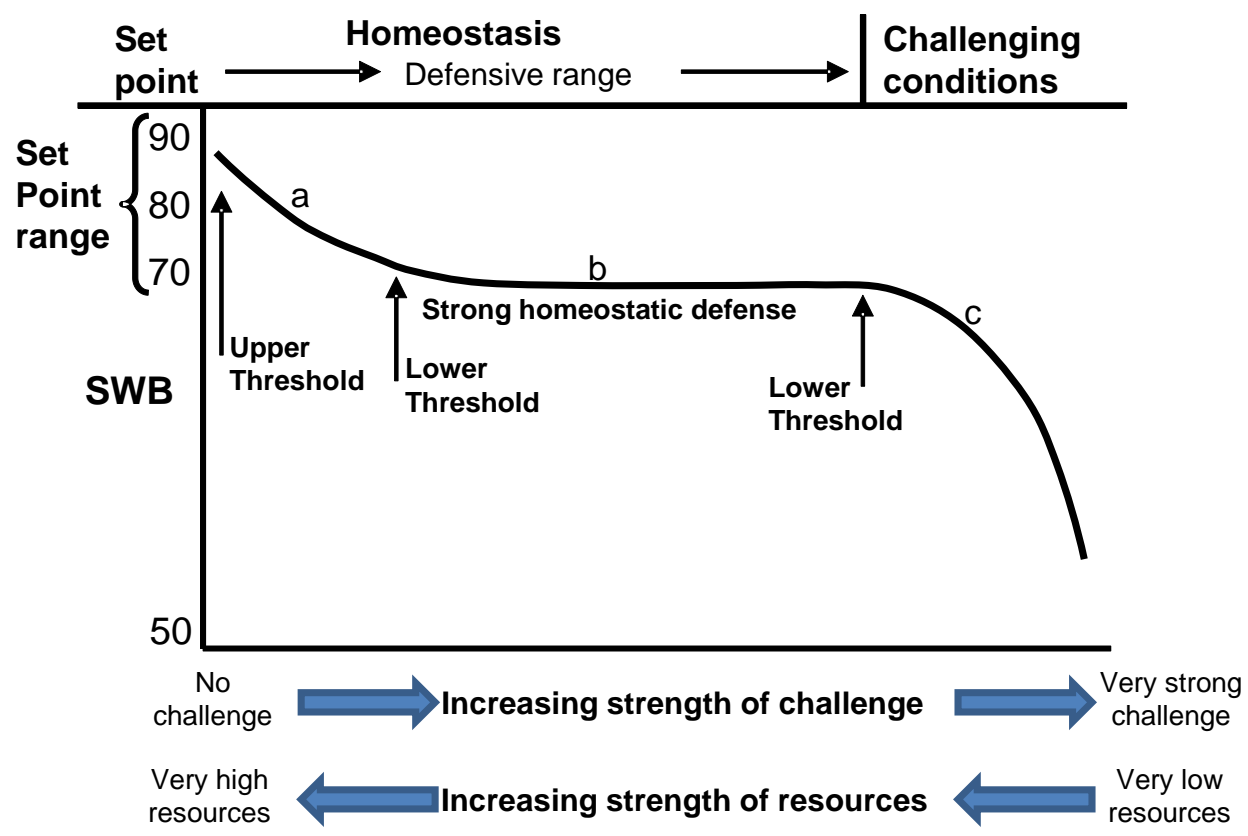

Figure 1: Changing levels of SWB as homeostasis is challenged. 


\section{University Library}

\section{- M M N E R VA A gateway to Melbourne's research publications}

Minerva Access is the Institutional Repository of The University of Melbourne

Author/s:

Cummins, RA;Wooden, M

Title:

Personal Resilience in Times of Crisis: The Implications of SWB Homeostasis and Set-Points

Date:

2014-02-01

Citation:

Cummins, R. A. \& Wooden, M. (2014). Personal Resilience in Times of Crisis: The Implications of SWB Homeostasis and Set-Points. JOURNAL OF HAPPINESS STUDIES, 15 (1), pp.223-235. https://doi.org/10.1007/s10902-013-9481-4.

Persistent Link:

http://hdl.handle.net/11343/282808 